\title{
Modelling and aggregation of heterogeneous fuzzy data
}

\author{
Arne-Jens Hempel Gernot Herbst Steffen F. Bocklisch
}

Chemnitz University of Technology, Germany

\begin{abstract}
This article proposes an individual fuzzy modelling and treatment of data allowing for the specific uncertainty of each datum. A modelling approach based on parametric fuzzy sets is being introduced which can be employed to model both data with individual uncertainties as well as abstract phenomena in a feature space (classes). An aggregation procedure is being presented which takes the individual characteristics of all learning objects into account, resulting in a fuzzy description of the same, interpretable structure. Examples are given using both synthetic and real-world data.
\end{abstract}

Keywords: Fuzzy classification, fuzzy modelling of data, aggregation.

\section{Introduction}

\subsection{Motivation for a fuzzy description of data and phenomena}

Perspective (I): acquisition of information. Information about a system or process might, for example, stem from experts of the respective field. Depending on the level of expertise, the information $x$ of every expert would be associated with an individual degree of uncertainty or vagueness reflecting this expert's experience or precision. Also, the quality of expert statements might vary with the manifestation of a given information (variable), i. e. dependent from a location in a feature space.

If information is given in form of linguistic variables or modifiers, e. g. "approximately $20 \mathrm{~cm}$ ", the interpretation of the uncertainty indicated by "approximately" by a precision engineer will probably be different from the interpretation of a home constructor. In another real-life example, the quantities in cooking recipes will be interpreted with varying precision depending on the experience of a professional or hobby cook. In the field of diagnosis of diseases, [1] reports on quantitatively different interpretations of linguistically identical assessments like "mild" or "severe".

In modelling such vague or uncertain expert statements, fuzzy sets have proven to be a useful tool [2].

On the other hand, when observing a system or process by measurements, the measured values are
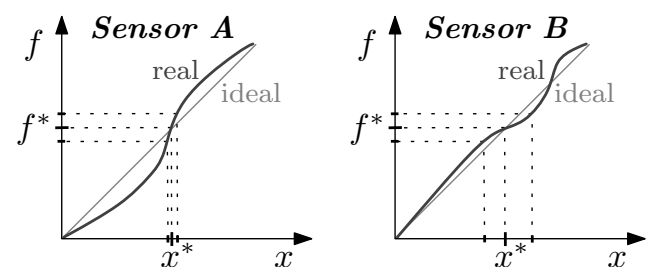

Figure 1: Identical readings $f^{*} \pm \Delta f$ of two sensors with different non-linear characteristics $f(x)$ may lead to values $x^{*}$ of the actual physical quantity with different uncertainty intervals. Also, the characteristics may change even for one sensor under different (non-stationary) measurement conditions.
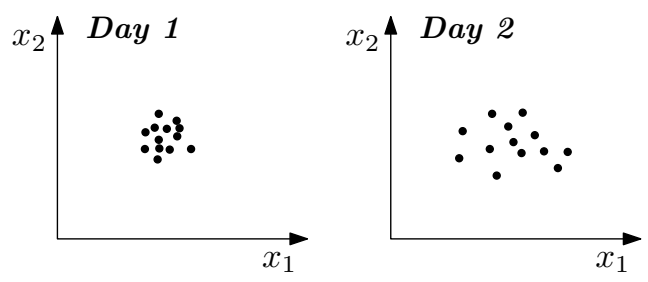

Figure 2: Series of position measurements made under different measurement conditions, influencing the uncertainty.

almost always afflicted with different kinds of uncertainty. If we, for instance, consider a sensor to measure a physical value which is of interest to us, the sensor output $f^{*}$ will most likely - dependent on the sensor principle and its realisation - not be directly proportional to the actual physical quantity $x^{*}$, but rather result of a non-linear dependency $f(x)$. If we consider two different sensors or sensor principles, an identical uncertain sensor reading $f^{*} \pm \Delta f$ will then translate into values of the actual physical quantity with different, asymmetric levels of uncertainty, cf. Fig. 1. The combination (fusion) of information from different sensors should therefore take the individual uncertainty of every measurement $x^{*}$ into account. Furthermore, sensor characteristics may change with time or conditions, such that precise values for the uncertainty interval cannot be obtained, and only a vague model for $f(x)$ could be determined.

When models for the non-linear characteristics of sensors are not available, one may conduct series of measurements $f$ to gain knowledge about the individual, asymmetric uncertainty of the desired physical value $x$. In addition, the uncertainty of 
measurements or measurement series may strongly depend on measuring conditions. For example, position measurements from radar or ultrasonic sensors might be influenced by weather conditions, cf. Fig. 2. Such a non-stationary behaviour of the uncertainty should also be treated and modelled individually.

Summarisingly, measurements or information about the same actual situation or even the same value of a parameter might be afflicted with varying, individual degrees of uncertainties. A precise specification the of interval borders for the uncertainty might, however, not always be available. For this reason, we advertise a fuzzy modelling of uncertainty intervals by means of fuzzy sets. While there are other powerful tools for the analysis of uncertain data available, fuzzy sets enable a joint modelling approach for both data- and expert-based information.

Perspective (II): classificatory modelling of phenomena. If we consider and observe a system with (one or several) characteristic phenomena in its behaviour, a model can-from an epistemological perspective - be created empirically from a set of data (observations or expert statements) [3]. If a recurring behaviour can be characterised by similar observations, a classificatory description of the system by means of classes in a feature space of the observations becomes feasible. That is each phenomenon can be assigned a certain (local) region of the feature space. Precise borders of such a region will, however, be difficult to determine from a limited set of uncertain measurements, cf. perspective (I). Subsequently, we will denote measurements, expert statements etc. as "objects" in a classificatory sense.

One uncertain object (one observation of a phenomenon-described in a fuzzy manner, as proposed above), may, of course, already constitute a class or atomic class on its own, as long as no further knowledge becomes available. This being said, a consistent and unified modelling of elementary observations (objects) and abstract descriptions of phenomena (classes) by means of fuzzy sets appears reasonable and will be proposed in this article.

\subsection{Aim and structure of this article}

As outlined in section 1.1, one main aim of this article is to provide a consistent fuzzy description of uncertain information (objects in a classificatory sense) on the one hand, and classes (i. e. models for characteristic phenomena) on the other. Section 2 will introduce a suitable means for the fuzzy description of multivariate data and classes.

Since a class may represent an abstract, aggregated form of the knowledge carried by the objects in a feature space, an aggregation procedure is required which transforms a set of heterogeneous

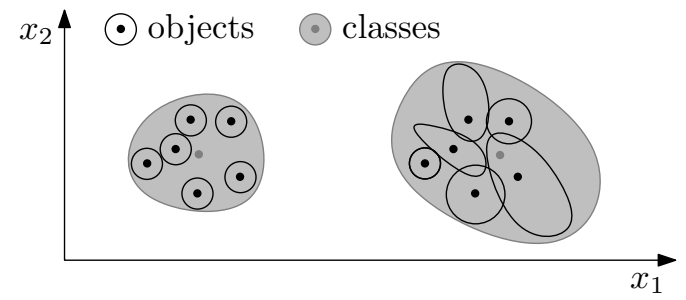

Figure 3: Aggregation of objects (black) with homogenous and heterogenous uncertainty to uncertain classes (grey).

fuzzy objects into an a fuzzy description of this class, taking the individual properties of every object into account, cf. Fig. 3. A procedure based upon the models from section 2 will be presented in section 3. Examples using synthetic as well as real-world data will be given in section 4 .

\section{Consistent modelling of fuzzy data}

In this section, we will present a consistent approach to fuzzy modelling of uni- and multivariate data using parametric fuzzy sets. The presented type of fuzzy sets will be employed both for modelling fuzzy objects (i. e. individual measurements or statements and their respective uncertainties) as well as aggregated classes of fuzzy objects (i. e. their abstract or combined description).

\subsection{A multivariate parametric fuzzy set}

Univariate basis function. Equation (1) defines an asymmetric fuzzy set $\mu: \mathbb{R} \mapsto(0, a]$ introduced by [4] as a generalisation of AIzERMAN's potential function.

$$
\mu(x)= \begin{cases}\frac{a}{1+\left(\frac{1}{b_{\mathrm{l}}}-1\right)\left(\frac{r-x}{c_{\mathrm{l}}}\right)^{d_{1}}}, & x<r \\ \frac{a}{1+\left(\frac{1}{b_{\mathrm{r}}}-1\right)\left(\frac{x-r}{c_{\mathrm{r}}}\right)^{d_{\mathrm{r}}}}, & x \geq r\end{cases}
$$

As visible in (1), this type of membership function is parameterisable by a set of seven parameters. Their effect shall be briefly described by means of Fig. 4. The maximum membership value $a$ of this unimodal function occurs at the modal point $r$, also denoted as the representative value of the set. For each of the left- and right-hand-side function branches, there is a set of parameters $b, c$ and $d$. The extents of the fuzzy set (in a crisp sense) is given by $c_{\mathrm{l}} / c_{\mathrm{r}}>0$, whereas the fuzziness is parameterised by $b_{\mathrm{l}} / b_{\mathrm{r}} \in(0,1]$ and $d_{\mathrm{l}} / d_{\mathrm{r}} \in[2, \infty)$, cf. Fig. $4 \mathrm{a}$ and $4 \mathrm{~b}$. Increasing values of $d$ cause sharper descents of the membership value to zero, and $d \rightarrow \infty$ will lead to rectangular (i. e. crisp) sets. 


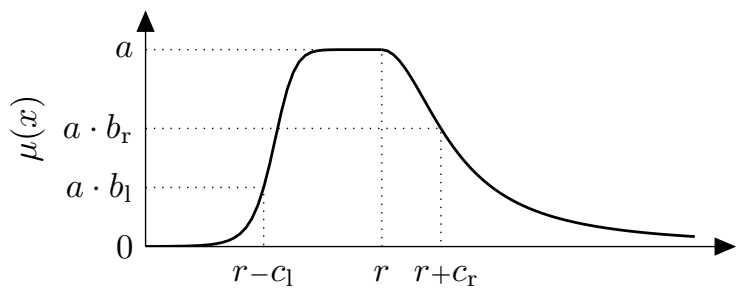

(a) Parameters $a, b, c$ and $r$

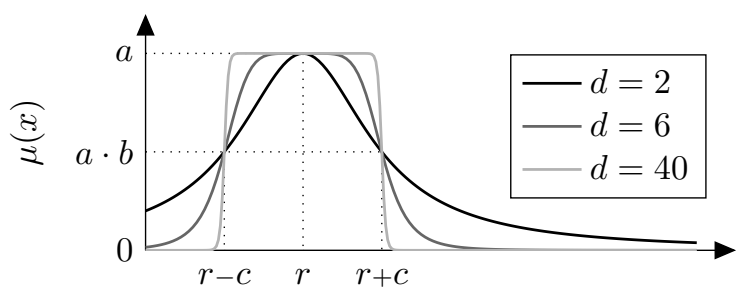

(b) $d$ parameters (here: $d_{\mathrm{l}}=d_{\mathrm{r}}=d$ )

Figure 4: Interpretation of the parameters in (1).

Extension to the multivariate case. For modelling multivariate data, we will need a multivariate membership function. Equation (1) can serve as a basis function to create such a multivariate set. For an $N$ dimensional membership function $\mu: \mathbb{R}^{N} \mapsto(0, a]$, we combine $N$ univariate normalised sets using a compensatory HAMACHER intersection (2) and assign a joint maximum membership value $a$ to the multivariate set [5]. The compensatory character of (2) guarantees interactivity of truth values while maintaining the desired border membership for $\boldsymbol{x}=\boldsymbol{r} \mp \boldsymbol{c}_{\mathbf{l} / \mathbf{r}}$, which would not be fulfilled by $t$-norm operators. The compensatory operator (2) satisfies all properties of an OWA operator [6].

$$
\cap_{\text {Ham }}^{N} \mu_{i}=\left(\frac{1}{N} \sum_{i=1}^{N} \frac{1}{\mu_{i}}\right)^{-1}
$$

The intersection in (2) can be interpreted analogously to the evaluation of a premise in rule-based fuzzy systems using multiple variables in a conjunctive combination:

$$
\mu(\boldsymbol{x})=\mu_{1}\left(x_{1}\right) \cap \ldots \cap \mu_{N}\left(x_{N}\right)
$$

As a benefit this approach, we obtain a parametric multivariate fuzzy set which exhibits the same interpretable structure as its univariate basis function $[4,7] .^{1}$ The resulting membership function is presented in (3) for the symmetric case. ${ }^{2}$ Figure 5 depicts two examples of asymmetric fuzzy sets in a two-dimensional feature space along with their uni-

\footnotetext{
${ }^{1}[8]$ employs different OWA and AIWA operators to combine membership functions of a similar parametric type, but does not obtain a parametric multivariate function.

${ }^{2}$ The asymmetric case can be obtained simply by using the appropriate left- or right-hand-side parameter set $\left(b_{\mathrm{l}, i}, c_{\mathrm{l}, i}, d_{\mathrm{l}, i}\right)$ or $\left(b_{\mathrm{r}, i}, c_{\mathrm{r}, i}, d_{\mathrm{r}, i}\right)$ for each dimension $i$ if $x_{i}<r_{i}$ or $x_{i} \geq r_{i}$, respectively.
}

variate basis functions for each dimension.

$$
\mu(\boldsymbol{x})=\frac{a}{1+\frac{1}{N} \sum_{i=1}^{N}\left(\frac{1}{b_{i}}-1\right) \cdot\left|\frac{x_{i}-r_{i}}{c_{i}}\right|^{d_{i}}}
$$

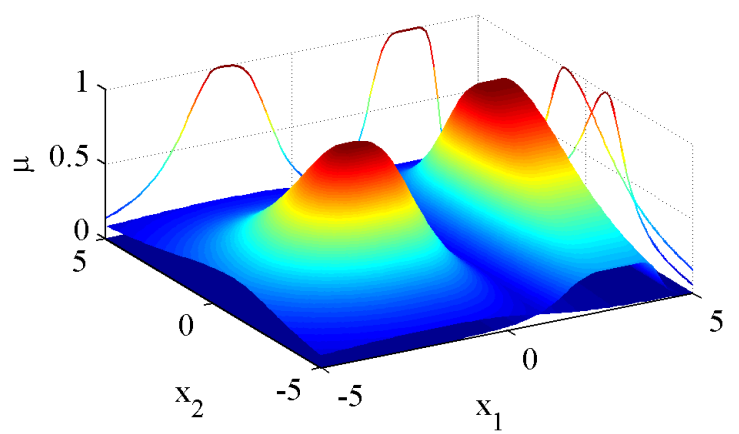

Figure 5: Two examples of two-dimensional sets.

Transformation of the feature space. In order to provide an even more "accurate" modelling of fuzzy data or phenomena, we will now allow our membership functions to be invididually rotated around their representative (modal) point $\boldsymbol{r}$ such that every membership function is defined in its own coordinate system centered at $\boldsymbol{r}$. The rotation will be carried out by $(N-1)$ subsequent rotations in the $\left(x_{1}, x_{k+1}\right)$-plane of the feature space using rotation angles $\varphi_{k}, k=1, \ldots, N-1$. The two-dimensional case is sketched in Fig. 6.

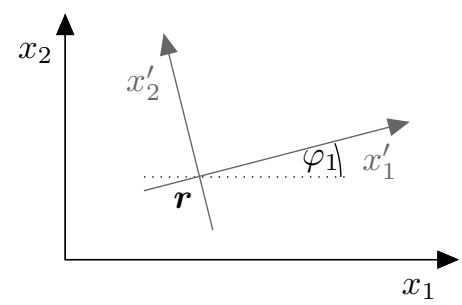

Figure 6: Transformation of the feature space for every membership function (translation $\boldsymbol{r}$ and rotation $\varphi$ ).

For every membership function, we will introduce an individual coordinate transformation

$$
\boldsymbol{x}^{\prime}=T(\boldsymbol{x}-\boldsymbol{r})
$$

where

$$
T=\left(\prod_{k=1}^{N-1} T_{k}^{\mathrm{T}}\right)^{\mathrm{T}}=T_{N-1} \cdot \ldots \cdot T_{k} \cdot \ldots \cdot T_{1}
$$

and for all $T_{k} \in \mathbb{R}^{N \times N}$ :

$$
T_{k}=\left(t_{i j}\right), t_{i j}= \begin{cases}\cos \varphi_{k} & , i=j=1 \text { or } k \\ \sin \varphi_{k} & , i=1, j=k \\ -\sin \varphi_{k} & , i=k, j=1 \\ 1 & , 1 \neq i=j \neq k \\ 0 & , \text { otherwise }\end{cases}
$$


Using the translation $r$ and the rotation matrix $T$ we can therefore compute the membership value for a rotated membership function by

$$
\mu(\boldsymbol{x})=\mu^{\prime}\left(\boldsymbol{x}^{\prime}\right)=\mu^{\prime}(T(\boldsymbol{x}-\boldsymbol{r}))
$$

with

$$
\mu^{\prime}\left(\boldsymbol{x}^{\prime}\right)=\frac{a}{1+\frac{1}{N} \sum_{i=1}^{N}\left(\frac{1}{b_{\mathrm{l} / \mathrm{r}, i}}-1\right) \cdot\left|\frac{x_{i}^{\prime}}{c_{\mathrm{l} / \mathrm{r}, i}}\right|^{d_{\mathrm{l} / \mathrm{r}, i}}}
$$

where the appropriate (left- or right-hand side) parameter set $\left(b_{\mathrm{l}, i}, c_{\mathrm{l}, i}, d_{\mathrm{l}, i}\right)$ or $\left(b_{\mathrm{r}, i}, c_{\mathrm{r}, i}, d_{\mathrm{r}, i}\right)$ for each dimension $i$ is selected if $x_{i}^{\prime}<0$ or $x_{i}^{\prime} \geq 0$, respectively. Figure 7 gives visual examples of the resulting membership functions.

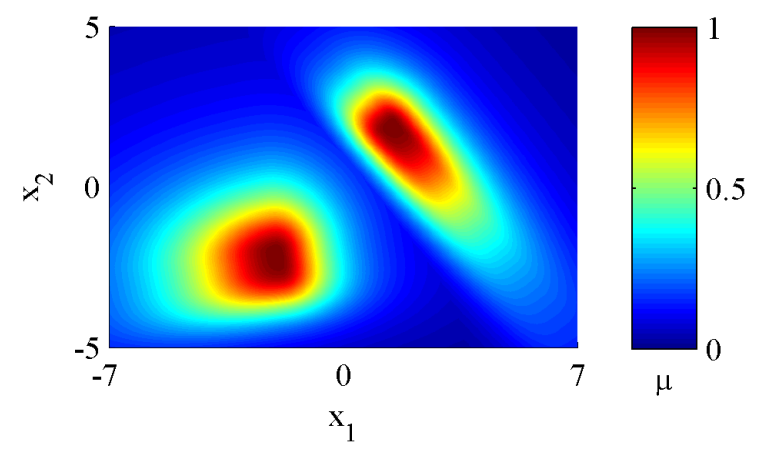

Figure 7: Two examples of individually rotated sets.

The (multivariate, parametric and rotated) fuzzy membership function presented in this section will form the unified basis of modelling fuzzy data and phenomena, regardless if individual data or aggregated, abstract phenomena are to be described.

\subsection{Fuzzy objects}

We subsume uncertain measurements or expertbased knowledge as objects in a feature space and propose their modelling by means of fuzzy sets. Equation (1) or its multivariate extension can be employed for this purpose in a straightforward and comprehensible manner. The parameters $r$ and $c_{\mathrm{l} / \mathrm{r}}$ define the representative (modal) value and the uncertainty interval, while $b_{1 / \mathrm{r}}$ and $d_{\mathrm{l} / \mathrm{r}}$ determine the fuzzy description of this uncertain information.

In many practical cases, however, detailed knowledge about the latter will probably not be available. In these cases, the definition of $b_{1 / \mathrm{r}}=0.5$ and $d_{\mathrm{l} / \mathrm{r}}=2$ has proven to be a reasonable choice [4]. For the normalised $(a=1)$ multivariate membership function its definition is being reduced to

$$
\mu(\boldsymbol{x})=\frac{1}{1+\frac{1}{N} \sum_{i=1}^{N}\left(\frac{x_{i}-r_{i}}{c_{\mathrm{l} / \mathrm{r}, i}}\right)^{2}}
$$

in the non-rotated case $\left(\varphi_{k}=0\right)$ and

$$
\mu^{\mathrm{o}}\left(\boldsymbol{x}^{\mathrm{o}}\right)=\frac{1}{1+\frac{1}{N} \sum_{i=1}^{N}\left(\frac{x_{i}^{\mathrm{O}}}{c_{\mathrm{l} / \mathrm{r}, i}}\right)^{2}}
$$

in the general case using an individual transformation $\boldsymbol{x}^{\mathrm{O}}=T_{\mathrm{obj}}(\boldsymbol{x}-\boldsymbol{r})$ into the object-specific coordinate system for each object. The position $r$ with the uncertainties $c_{\mathbf{l} / \mathbf{r}}$ and the rotation angles $\varphi_{k}$ remain as free parameters to individually model a fuzzy object in its feature space.

The latter may be of importance especially for a more precise modelling of expert statements, i. e. regions of the feature space. When dealing with uncertain measurements or data, the rotation angles will usually remain $\varphi_{k}=0$ as long as no further information about the measurement process becomes available.

\subsection{Fuzzy classes}

For an abstract, aggregated knowledge representation of a phenomenon, (1) and its multivariate extension may equally well be used to model a region in the feature space with soft borders. If this class stems from an aggregation of fuzzy objects, i.e. an individually fuzzified description of a set of data, it appears reasonable that a higher degree of customisation compared to section 2.2 will be necessary to capture the aggregated knowledge in an adequately detailed manner. In contrast to section 2.2 , we therefore recommend to employ all parameters available in (1). The rotation angles of the class specific coordinate system can furthermore be used to represent correlations between individual features for this particular class or phenomenon, cf. Fig. 7 .

Since the underlying compensatory HAMACHER operator (2) satisfies the properties of an OWA operator, the aggregated fuzzy representation of multivariate data forms a compromise of the different features supporting this class.

\section{Aggregation of fuzzy objects}

Knowledge about uncertainties of measurements (objects) may often be gained from the measurement process or an expert. In order to derive an abstract description of a phenomenon-i.e. a class in the feature space - based upon elementary measurements or objects, an aggregation procedure is required which takes the individual uncertainties of measurements into account. If the individual objects are being modelled by means of section 2.2, such an aggregation procedure has to transform a set of multivariate parametric membership functions into one membership function of this type describing the resulting class.

In contrast to non-parametric methods such as kernel density estimation, our proposed approach 
again results in a parametric model, i.e. the parametric description of $M$ objects is being mapped to only one resulting set of class parameters. Such a compression of information, of course, means that the resulting model can only be an approximation of the input data set. On the other hand, the parametric model is well-interpretable (cf. section 2.1) and may provide a significant reduction of data.

In [7] a suitable object-specific approach to this problem was being presented based on [4], where only feature/class-specific uncertainties could be treated. We will pick up and further extend this approach in order to aggregate fuzzy objects with individual rotation in the feature space. In the following, we will briefly describe the procedure in a condensed and visual manner. ${ }^{3}$

In order to distinguish the variables and parameters involved, all coordinates belonging to an object or the resulting class are denoted by a subscript "obj" or "class", respectively. If a parameter or coordinate is being defined in a object- or class-specific coordinate system, it is furthermore being specified by a superscript "o" or "c".

Step (1): Adjust object coordinates. As the representative value of the class $\boldsymbol{r}_{\text {class }}$ shall be interpretable as the centre of mass, the objects' individual centres of mass are being approximated ${ }^{4}$ in their respective coordinate system by weighing their extents $c_{\mathrm{obj}, 1 / \mathrm{r}, i}$ with the border memberships $b_{\mathrm{obj}, 1 / \mathrm{r}, i}$ in each dimension $i$, resulting in the adjusted representative value $\tilde{\boldsymbol{r}}_{\mathrm{obj}}^{\mathrm{o}}$. Since, per convention from section 2.2, the border memberships of objects are set to $b_{\mathrm{obj}, 1 / \mathrm{r}, i}=0.5$, the computation for each object is being reduced to

$$
\tilde{r}_{\mathrm{obj}}^{\mathrm{o}}=\left(\begin{array}{c}
\vdots \\
-0.5 \cdot c_{\mathrm{obj}, 1, i}+0.5 \cdot c_{\mathrm{obj}, \mathrm{r}, i} \\
\vdots
\end{array}\right),
$$

which is then transformed back into the feature space, yielding $\tilde{\boldsymbol{r}}_{\mathrm{obj}}$ :

$$
\tilde{\boldsymbol{r}}_{\mathrm{obj}}=T_{\mathrm{obj}}^{-1}\left(\tilde{\boldsymbol{r}}_{\mathrm{obj}}^{\mathrm{o}}\right)+\boldsymbol{r}_{\mathrm{obj}}
$$

Step (2): Compute the class centre. The representative (modal) value of the resulting fuzzy set $\boldsymbol{r}_{\text {class }}$ is being set to the mean value of all objects' adjusted positions, $M$ being the number of objects,

$$
\boldsymbol{r}_{\text {class }}=\frac{1}{M} \sum_{k=1}^{M} \tilde{\boldsymbol{r}}_{\mathrm{obj}, k}
$$

so that the resulting $\boldsymbol{r}_{\text {class }}$ can be interpreted as the centre of mass of all fuzzy objects, cf. Fig. 9.

\footnotetext{
${ }^{3} \mathrm{~A}$ more detailed description is available in [7].

${ }^{4}$ The precise computation of an object's centre of mass would, of course, be possible, but require the numerical solution of an integral.
}

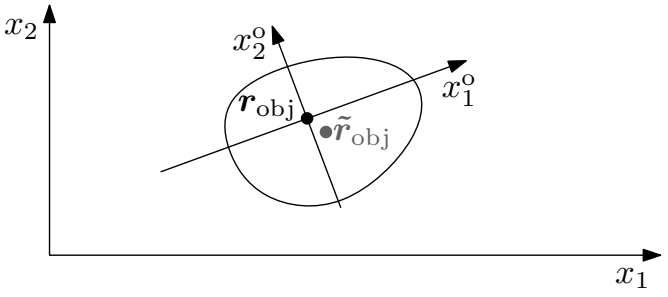

Figure 8: Aggregation, step (1): Adjusting the position $\boldsymbol{r}_{\text {obj }}$ (black) of each object to its centre of mass $\tilde{\boldsymbol{r}}_{\text {obj }}$ (grey).

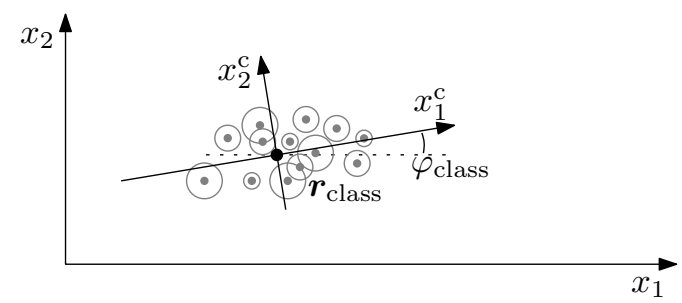

Figure 9: Aggregation, steps (2) and (3): Compute the aggregated centre of mass $\boldsymbol{r}_{\text {class }}$ from all objects (grey) and the class rotation.

Step (3): Compute the class rotation. The classspecific coordinate system is then being rotated around its centre $\boldsymbol{r}_{\text {class }}$ by $N-1$ rotation angles $\varphi_{\text {class, } k}$ such that the axes become the neutral axes through the set of objects represented by their adjusted positions $\tilde{\boldsymbol{r}}_{\mathrm{obj}}^{\mathrm{o}}$ (comparable to a principal component analysis), cf. Fig. 9. From the rotation angles, the class-specific rotation matrix $T_{\text {class }}$ can be obtained via (5).

Step (4): Transform objects into class space. Before the shape of the class can be determined, all objects have to be transformed into the class-specific coordinate system. At first, the adjusted representative value of each object $\tilde{\boldsymbol{r}}_{\text {obj }}$ is being transformed:

$$
\tilde{\boldsymbol{r}}_{\mathrm{obj}}^{\mathrm{c}}=T_{\text {class }}\left(\tilde{\boldsymbol{r}}_{\mathrm{obj}}-\boldsymbol{r}_{\text {class }}\right)
$$

In order to aggregate all objects based on their parameters, we need these parameters of the objects' membership functions in the class-specific coordinate system. The parametric membership function defined in each object's coordinate system, however, cannot be transformed to the class-specific coordinate system remaining in its original analytical form (1). We therefore approximate the extents of the transformed membership function by determining the piercing points $\tilde{c}_{\mathrm{obj}, 1 / \mathrm{r}}$ of the class-specific unity vectors with the alpha cuts of an object, cf. Fig. 10. Due to the choice of parameters $b$ and $d$ in section 2.2, equations for the alpha cuts are available in form of ellipses in each quadrant, so that the approximated $\tilde{c}_{\mathrm{obj}, 1 / \mathrm{r}}$ can be obtained without difficulty from the $c_{\mathrm{obj}, 1 / \mathrm{r}}[9]$.

Step (5): Compute class extents. When the centre of the class, its specific coordinate system and 


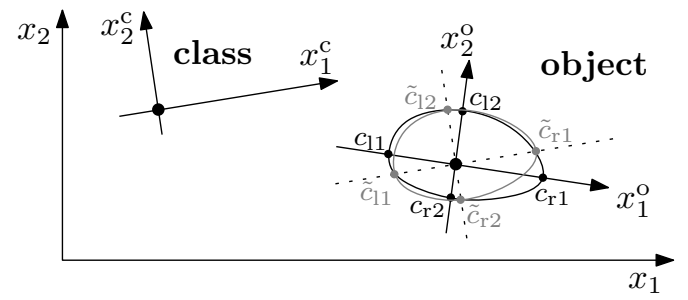

Figure 10: Aggregation, step (4): Approximation (grey) of the object's extents (black) in the class space by computing piercing points of the class unity vectors with the object's alpha cuts.

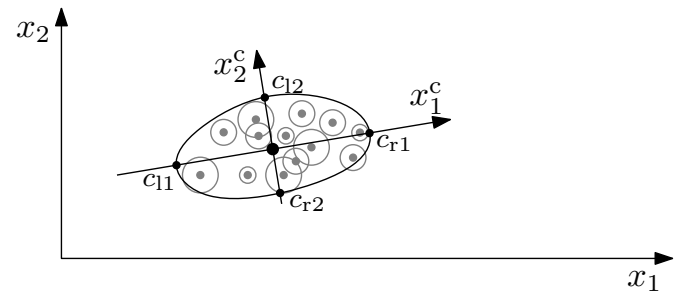

Figure 11: Aggregation, step (5): The extents of the class $\boldsymbol{c}_{\text {class }, 1 / \mathrm{r}}$ are specified by the outermost objects and their extents.

the transformed objects are available, the extents of the class $\boldsymbol{c}_{\text {class }, 1 / \mathrm{r}}$ can be determined as follows, cf. Fig. 11:

$$
\begin{aligned}
& \boldsymbol{c}_{\mathrm{class}, \mathrm{l}}=\min _{k \in\{1, \ldots, M\}}\left(\tilde{\boldsymbol{r}}_{\mathrm{obj}, k}^{\mathrm{c}}-\tilde{\boldsymbol{c}}_{\mathrm{obj}, k, \mathrm{l}}^{\mathrm{c}}\right) \\
& \boldsymbol{c}_{\mathrm{class}, \mathrm{r}}=\max _{k \in\{1, \ldots, M\}}\left(\tilde{\boldsymbol{r}}_{\mathrm{obj}, k}^{\mathrm{c}}+\tilde{\boldsymbol{c}}_{\mathrm{obj}, k, \mathrm{r}}^{\mathrm{c}}\right)
\end{aligned}
$$

Step (6): Determine softness. In each dimension $i$, the set of objects is being divided into subsets to the left and to the right of the representative value of the class $r_{\text {class }, i}$. The distribution of each subset is being compared with a geometric series. In this case, the parameter $d_{\mathrm{l} / \mathrm{r}, i}$ of the class is being assigned a value close to $d_{1 / \mathrm{r}, i} \rightarrow 2$ (soft borders). If, on the other hand, the objects' distribution rather resembles a uniform distribution (or accumulates at the class borders), the fuzzy set will become more crisp by chosing higher values for $d_{\mathrm{l} / \mathrm{r}, i}$, cf. Fig. $4 \mathrm{~b}$ and [4].

Step (7): Determine border membership. As the final parameter, the border memberships of the class $b_{\mathrm{l} / \mathrm{r}, i}$ have to be determined in each dimension $i$. As described in [4] and [7], the values for $b_{\mathrm{l} / \mathrm{r}, i}$ are chosen such that the cardinality of the aggregated set preserves the weight of the objects:

$$
\int \mu_{\text {class }}\left(x_{i}\right) \mathrm{d} x_{i} \stackrel{!}{=} \int \max _{k \in\{1, \ldots, M\}} \mu_{\mathrm{obj}, k}\left(x_{i}\right) \mathrm{d} x_{i}
$$

\section{Examples}

\subsection{Synthetic data}

As a first example, we will demonstrate the effects of heterogeneously described fuzzy objects on the outcomes of the aggregation procedure from section 3 . Figure 12 depicts two datasets with an identical object distribution (black), albeit different uncertainties (grey). The lower set of data features individual, asymmetric values for each object's uncertainty.

While the actual results of the aggregation procedure according to section 3 are sets of parameters for the membership function of section 2.1, we will restrict ourselves to their visual presentation, which is more intuitively comparable.

The results of the aggregation steps (i.e. fuzzy membership functions) for each dataset are visible in Fig. 12 by the colour shading. One can observe that the individual uncertainties of the objects influence all parameters of the class. The modified weights of the objects - especially the outermost objects - in the lower dataset result in a different rotation of the lower class. Furthermore, the adapted extents and fuzziness (softness) are well visible.

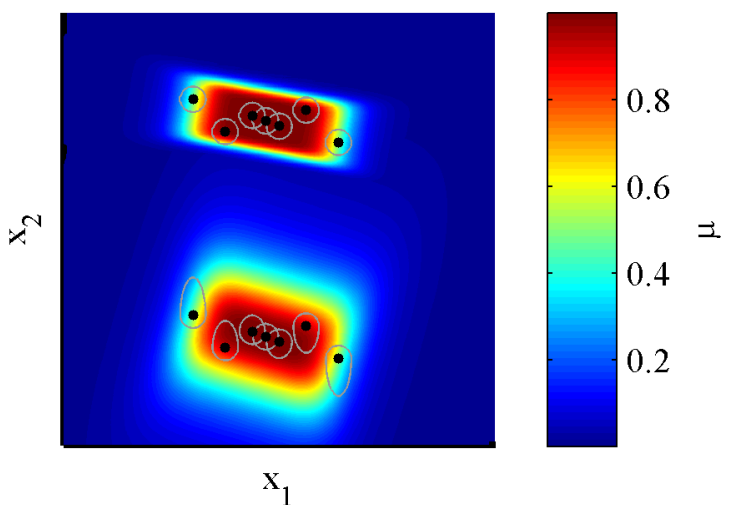

Figure 12: Synthetic data. Two sets of objects (black) with homogeneous and heterogenous uncertainties (grey) along with the aggregated fuzzy class description.

\subsection{Real-world data}

As a second example, we will aggregate objects from a real-world dataset, originating from a geodetic observation network that monitors the continental drift in Iceland [10]. The feature space of these data consists of the movement magnitude of reference points (measured by GPS over the years 1993-2004) and their direction.

[11] determined individual uncertainties for each object, i. e. both measurements and expert knowledge were incorporated into the description of each object. Due to the parametric approach and its interpretability, the proposed fuzzy model from section 2 can be employed without difficulty in this hy- 


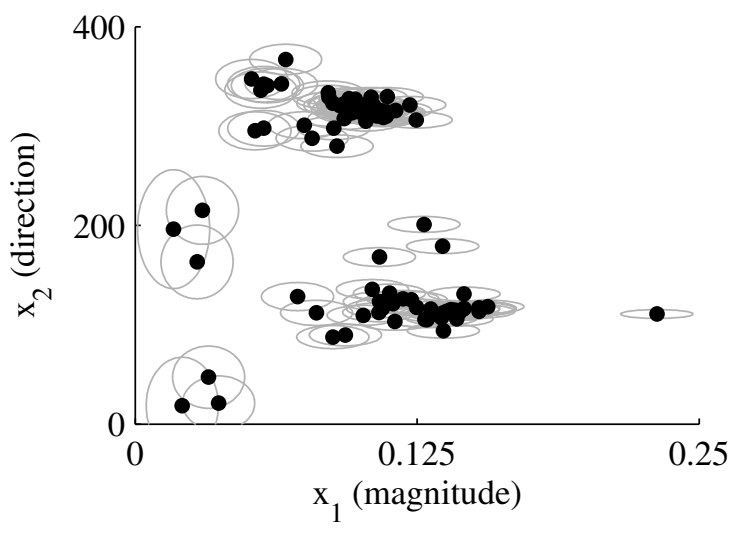

Figure 13: Isnet dataset. The features are: movement magnitude (in metres) and direction (in gon), their individual uncertainties are marked by ellipses.

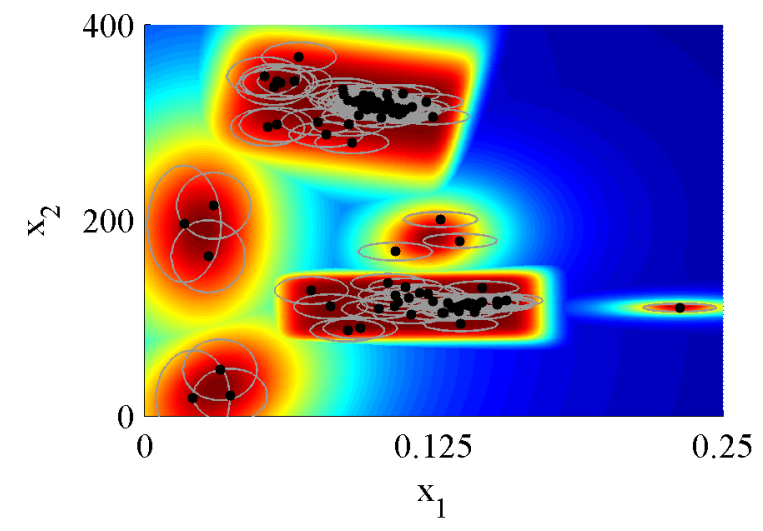

Figure 14: Aggregated fuzzy class description of the data from Fig. 13.

brid case. ${ }^{5}$ The data are depicted in Fig. 13 along with their individual uncertainties.

Based on domain-specific expert knowledge, movement patterns (classes of objects) can be identified in this dataset in form of six clusters [11]. Aiming at an abstract knowledge representation of these patterns (classes), an aggregated description can be found using the methods described in section 3 .

As in section 4.1, we will again restrict ourselves to a visual presentation of the results. However it has to be mentioned that also a significant data reduction was achieved, namely from the fuzzy description of 115 objects to the parameter sets of six aggregated models.

The results of the aggregation procedure incorporating the individual uncertainties of the data from Fig. 13 are presented in Fig. 14. As can be seen on the very right of Fig. 13, one class description has been obtained from a single fuzzy object, forming an atomic class on its own. Furthermore it can be observed that the influence of individual properties

\footnotetext{
${ }^{5}$ The parameters $r$ contain the object positions from the measurements, whereas the parameters $c_{1 / r}$ express the uncertainty based on expert knowledge.
}

of objects is stronger in classes with fewer objects, e. g. the two classes on the lower left side.

Comparision to a non-parametric method. As mentioned in section 3, an aggregation with parametric output entails a certain loss of information. We will therefore compare our results of Fig. 14 with a standard kernel density estimation (KDE) for each of the clusters of objects from Fig. 13, which should allow for a more detailed representation of the original data. The bandwidth was determined by errorminimisation (AMISE) for each cluster.

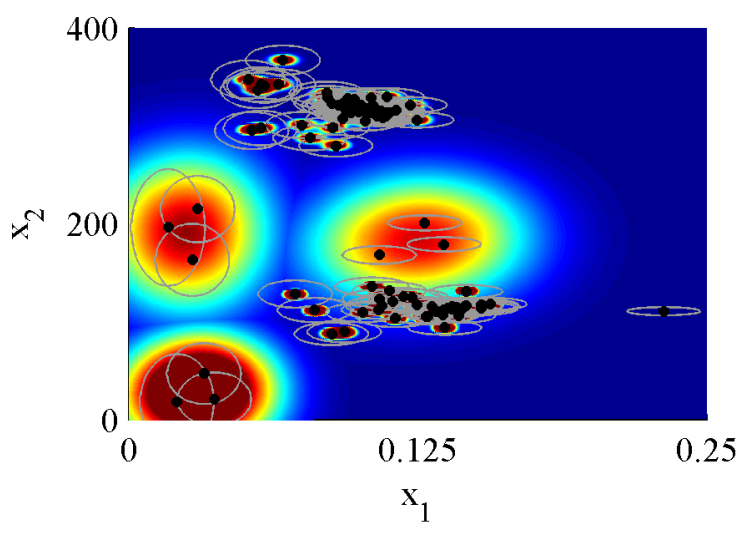

Figure 15: Kernel density estimation for each cluster of objects from Fig. 13. Warmer colours represent higher densities.

The density estimation for each cluster is shown in Fig. 15, along with the original objects. ${ }^{6}$ In comparison to Fig. 14, one may notice that single objects form atomic classes in Fig. 14, but are treated as outliers by the KDE. ${ }^{7}$ The shape of the three classes with three objects in Fig. 14 is better adapted to the uncertainty of their objects due to the class rotation. The main difference, however, can be observed in the two densely-populated clusters. The multimodal KDE results in Fig. 15 form a non-contiguous density model, while the original uncertain data occupy an almost contiguous area of the feature space. In Fig. 14, each aggregated class model comprises all of its supporting objects by one unimodal membership function. Despite the significant data reduction, the loss of information inherent to the parametric model in Fig. 14 appears negligible except for the uppermost class.

Apart from the visual comparison for this example, however, a main feature of the approach presented in this article is the compact parametric form of the aggregation results. These can seamlessly be employed to create well-interpretable classifiers or knowledge bases.

\footnotetext{
${ }^{6}$ It has to be noted, however, that a standard KDE cannot incorporate the individual uncertainty of each object.

${ }^{7}$ Depending on the application, either approach may be an advantage or disadvantage.
} 


\section{Conclusion}

As the uncertainty of a given set of data may not always be precisely known, fuzzy sets provide one possible approach to model uncertain data, especially when dealing with both data-based and expertbased information. For different reasons, the uncertainty may furthermore vary for each datum. This behaviour could also be observed in a real-world example in this article.

For the case of data with heterogeneous uncertainty, a modelling approach based upon multivariate parametric fuzzy sets was presented in this article in order to describe both data and aggregated data (classes) in a consistent manner. One principle of this approach is that a single measurement or expert opinion may already be representative for a certain phenomenon, i. e. form a class on its own. Therefore the same modelling tools should be employed both for data and aggregated data (classes).

An aggregation procedure was introduced which takes the individual uncertainty of data points into account, while maintaining the parametric modelling approach. As shown in a synthetic and a realworld example, the presented approach is able to capture the individual characteristics of single objects (measurements) in the aggregated fuzzy representation.

\section{References}

[1] Mila Kwiatkowska, Krzysztof Kielan, and Krzysztof Michalik. A fuzzy-semiotic framework for modeling imprecision in the assessment of depression. In Joao P. Carvalho, Didier Dubois, Uzay Kaymak, and Joao M. C. Sousa, editors, 2009 International Fuzzy Systems Association World Congress and 2009 European Society for Fuzzy Logic and Technology Conference (IFSA-EUSFLAT 2009), pages 17171722, 2009.

[2] Lotfi A. Zadeh. The concept of a linguistic variable and its application to approximate reasoning-II. Information Sciences, 8(4):301$357,1975$.

[3] Hans-Jürgen Zimmermann. Fuzzy Set Theory and its Applications. Springer, 2001.

[4] Steffen F. Bocklisch. Prozeßanalyse mit unscharfen Verfahren. Technik, Berlin, 1987.

[5] Ullrich Scheunert. Fuzzy-Mengen-Verknüpfung und Fuzzy-Arithmethik zur Sensor-DatenFusion. PhD thesis, TU Chemnitz, 2001.

[6] Ronald R. Yager. On ordered weighted averaging aggregation operators in multicriteria decisionmaking. IEEE Transactions on Systems, Man and Cybernetics, 18:183-190, January 1988.

[7] Arne-Jens Hempel and Steffen F. Bocklisch. Fuzzy pattern modelling of data inherent structures based on aggregation of data with het- erogeneous fuzziness. In Gregorio Romero Rey and Luisa Martinez Muneta, editors, Modelling Simulation and Optimization, chapter 28, pages 637-655. INTECH, 2010.

[8] Uwe Mönks, Denis Petker, and Volker Lohweg. Fuzzy-pattern-classifier training with small data sets. In Eyke Hüllermeier, Rudolf Kruse, and Frank Hoffmann, editors, Proceedings of the 13th International Conference on Information Processing and Management of Uncertainty (IPMU 2010), volume 80 of Communications in Computer and Information Science, pages 426-435, Heidelberg, 2010. Springer.

[9] Michael Päßler. Mehrdimensionale Zeitreihenmodellierung und -prognose mittels Fuzzy Pattern Modellen. Diplomarbeit, TU Chemnitz, 1998.

[10] Guðmundur Pór Valsson, Pórarinn Sigurðsson, Christof Völksen, and Markus Rennen. ISNET2004: Niðurstöður úr endurmælingum grunnstöðvanets islands. Technical report, Landmælingar Islands, 2007.

[11] Rainer Fletling. Methodische Ansätze zur unscharfen Mustererkennung bei Deformationsmessergebnissen. PhD thesis, TU Braunschweig, 2010. 